\title{
The gMenu User Interface for Virtual Reality Systems and Environments
}

\author{
Andrew Dunk and Adrian Haffegee \\ Centre for Advanced Computing and Emerging Technologies \\ The University of Reading \\ a.dunk@reading.ac.uk \\ http://www.acet.reading.ac.uk
}

\begin{abstract}
Desktop computers are able to provide a user interface with many features that allow the user to perform tasks such as execute applications load files and edit data. The gMenu system proposed in this paper is a step closer to having these same facilities in virtual reality systems. The gMenu can currently be used to perform a selection of common tasks provided by a user interface, for example executing or closing virtual reality applications or scenes. It is fully customisable and can be used to create many different styles of menu by both programmers and users. It also has shown promising results bringing some of the system based commands into the virtual environment, as well as keeping the functionality and adaptions required by applications. The use cases presented demonstrate a collection of these abilities.
\end{abstract}

Keywords: 3D User Interfaces, Virtual Reality.

\section{Introduction}

The development of quality 3D user interfaces and virtual environments can be complicated and time consuming. Currently there are no recognised standards on which to base the design and implementation of 3D user interfaces. Questions regarding interaction techniques, such as selections, travelling and way finding may have been answered already [1]. The area of environment and system control however, has not been paid as much attention.

Every computer needs an operating system that allows the user to utilise the computer hardware in an efficient way. These operating systems are combined with user interfaces to enhance its usability and usefulness. This research intends to be a step towards a three dimensional operating system for virtual environments. It proposes combining a customisable menu system, currently being developed as part of this research, with a virtual reality frameworks such as VieGen [2] which is currently being used for testing the gMenu system. This combination will supply equivalents for the more common operations provided by today's desktop operating systems. These will include simple file browsing, application execution, system controls (such as audio settings), as well as being able to perform basic object manipulations within an environment (including scaling, rotations, and constraining movement to a specific axis). 
This paper starts by introducing a background on existing user interfaces, then continues by elaborating on the gMenu's current and proposed features. Several use cases are described, and finally some conclusions are drawn and future work is proposed.

\section{Virtual Reality User Interfaces}

In order to compile a standard for 3D user interfaces, studies need to be made on existing 3D user interfaces which have been designed for specific applications, and have gradually improved over time as new requirements and issues where found. There has been significant research into comparing and classifying these user interfaces to gain a overall understanding of the requirements of a useful and usable user interface. 113.

\subsection{Interaction Techniques}

There are many interaction techniques available for 3D user interfaces that allow users to navigate through environments, as well as select and manipulate objects.

Selection is an integral part of using a menu and there are many different methods available for this; for example, collision detection is one of the simplest methods of selection, the user would reach out and touch the object to select it. This becomes difficult if the objects are placed at further distances. To overcome this ray casting selection could be used; this method simulates a laser pointer extending the selection to any distance.

Other selection methods include occlusion which works by concealing the object to be selected. This method is relative to your eye and hand position. 4] There are also selection techniques that use eye tracking only, enabling selections to be made based on the users gaze rather than their hand position. [5]

\subsection{System and Environment Control Menus}

3D menu systems exist in many forms and designs. Types of menus include 2D menu systems that have been brought into 3D environments, 3D menus positioned at specific locations relative to the environment, the users, or objects, and menus that are specific to hardware devices used with the virtual reality system.

There are many 2D graphical user interfaces available, one solution is to use these systems and adapt them to the 3D environment. This gives advantages to usability as systems like Windows Icons Menus and Pointers (WIMP) have had many years of research and improvements applied to them. They are very well recognised and would require a minimal amount of training for users to confidently use them.

A variety of pop-up and pull-down style menus have been designed and studied in virtual environments [6], as well as implementations enabling the $\mathrm{X}$ window system to be brought into a virtual environment [7. Menus have also been defined 
by their position in the environment, the tool belt menu was displayed around the users waist while in the environment and hand held menus such as the ring menu is displayed by the users hand while being used.

A multitude of menus have been designed for use with particular hardware in mind like the TULIP menu [8] which use pinch gloves. Menus can be projected onto physical objects like tablets which in turn give a tactile feedback as well as visuals when being used. 9

Other menu systems include the Spin Menu 10, designed for quick access and uses 3D icons and the Command and Control Cube 11 used on the holobench. These two menus use an action or movement to select menu items rather than the idea of pointing and selecting.

\section{The gMenu System}

This section is split into three parts. The first describes some of the proposed and current abilities of gMenu and how it can be constructed and configured. The second describes the menu as a file browser and how it is able to create icons for specific file types. The third section describes messaging between the menus, objects within a scene, and the virtual environment in conjunction with the VieGen framework.

\subsection{A gMenu}

Each new menu is constructed with a menu grid, which is made up of multiple menu items. It is possible to create new menus within an existing menu producing submenu functionality. Submenus automatically link back to their parent when created.

Multiple gMenus can be created in a virtual environment, each gMenu could be organised to give better clustering of the commands used within the virtual environment.

The menus will be able to be created by programmers and customised by users. Simple XML files will be able to be written by users to generate custom menus and link there functionality to the system and environment. An XML parser will be used to generate the user created menus and bring them into the environment. The programmer will have a powerful library to configure all aspects of a created gMenu.

gMenu Items. gMenu items are created and inserted into locations on a gMenu grid. It is possible to develop new gMenu items by extending a base gMenu item container. Currently there are three types of items that are available, these are a textured button which is a cuboid object with textured surfaces, a 3D item which is a detailed 3D model object rather than an flat texture, and a 3D text string for displaying text within the menu.

Each item has some base properties including its position in the grid, its visibility, if it is enabled, highlighting function, states, and messaging options 


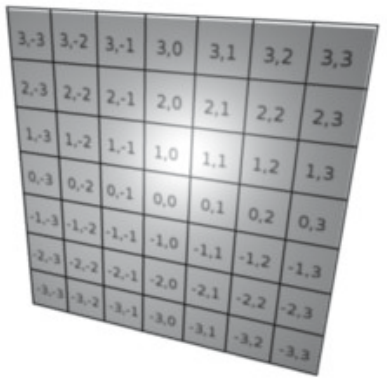

(a) Flat Plane Grid

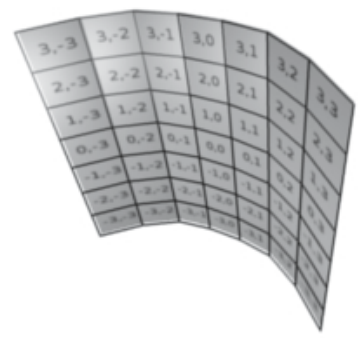

(b) Curved Wall Grid

Fig. 1. Examples of Possible Grid Positioning with Associated Coordinates

for communicating the states and commands throughout the system. When a menu is being displayed within an environment, each of the items are frequently checked for collisions with the users interface device. Items are able to send messages describing their current state. for instance if the user is hovering over the item, pressing a button on the users interface device over an item, or releasing a button on the users interface device while over an item.

If a hover state is triggered then a highlighting function is applied to the item. The default highlight translates the item forward towards the user by the same depth as the item, giving the effect of the button being selected. However the highlight functions can be replaced allowing the creator to develop their own customised functionality.

The gMenu Grid. The gMenu grid is designed to give the items created within the menu a defined structure and keep the visual aspect of the menu clean. This allows the creator to concentrate on the functionality of the menus rather than their appearance.

The grid is never displayed and only exists as a function that generates a position in $3 \mathrm{D}$ space for a given $(x, y)$ item coordinate. To place an item into the grid simply give the item an $(x, y)$ coordinate, which is translated into the position of the item in 3D space. This also allows new grid generation functions to be written, meaning the menus physical shape and style can be customised. A flat plane Fig 1(a) or a curved wall Fig 1(b) are possible examples of grids that can be created for positioning each of the items. Items do not have to exist on a single plain but they are dependant on the grid. Items can be placed at any location within the grid allowing items to be clustered and arranged into sections and different shapes Fig 2(b).

The size of the cells in the grid and their separation can be set when creating the menu grid, this denotes the maximum size of the menu items and the distance they are placed apart from one and another. The grid itself can be as large as 
required. However considerations need to be made if creating large grids, at the efficiency of searching for an item within a large grid could possibly produce poor results.

\subsection{The gMenu File Browser}

The gMenu file browser extends the functionality of the base gMenu system to create an iconic display of a file system starting from a root directory.

When a gMenu file browser is created a recursion of the file system is performed, each file and folder beneath the root path is read and an item is created within the file browser grid. Submenus are created for each directory with all appropriate files included as separate items. As each item is added to the menu a textured button is created and supplied a texture to represent the file or folder. In addition a text item is created to identify each icon by displaying the file or folder name.

Some file types are recognised by the file browser, these are given specific icons. If supported image files are found within the file system the image will be used as the buttons texture. Recognised 3D model files are either added to the menu as a scaled version of the 3D model, or a textured button can be displayed with an image created from the 3D model file. By creating images for the 3D models the system load is reduced. Any model images created are saved in the same directory as the 3D model to decrease load times on consecutive runs. There is also support for basic filtering of file extension types, making it possible to create file browsers of specific file types only. If a folder is selected the corresponding submenu will replace the current menu, if an item is selected, the items file name and location are returned.

Creating Button Textures. If a button texture is needed for a $3 \mathrm{D}$ model a set of steps are taken. Firstly a search is performed in the same directory as the file for a previously created texture associated with the file, if one is found that texture is simply returned as the files icon. If a texture file is not found then a new one is created.

This is performed by loading the model into a predefined scene that is never rendered on the users display. The scene has some basic settings that can be customised, such as the size of the viewport being used which determines the end size if the icon, and the scenes background colour. Once the 3D model has been loaded into the scene a camera is positioned to capture the entire model and an image is rendered and saved in the same directory as the model so that the texture will not need to be generated again, saving load time. Once the image has been saved it is returned to be used in the menu item.

\subsection{VieGen Specific Implementation}

Within VieGen, the different entities that make up a virtual environment are all derived from the base SceneObject class, which provides them with in-scene 
attributes and behaviours. All SceneObjects have the ability to produce and consume messages, which are used to provide communication and interaction amongst these entities. Typical VieGen messages include those such as the user selecting an object, or notification that one object has collided with another. These messages provide the basis for interacting with the gMenu system. Focus changing or selection events can be used to indicate changes in the gMenu Item state, and the generation of new messages could result from these interactions. While some items could be hard coded to provide specific actions, others could be configurable. It the latter case, options could range from sending named system events, through to the flexibility of sending XML fragments for direct object control.

\section{Applications}

This section describes some simple applications using the gMenu and shows its current functionality.

\subsection{D Model Viewer}

To demonstrate the gMenus file browser features, a simple environment has been created that allows users to brows through a file hierarchy and load selected 3D models into the environment, position the models within the environment and change their orientation. The environment itself is a small area with no visible walls or floor, it contains four lights to equally illuminate the area and has a blue background colour.

The gMenu file browser can be opened in the environment by pressing a button on the users wand. The menu appears a short distance in front of the wand position and a ray casting technique is used to select items within the menu. The file menu is filtered to only show supported 3D model files. Textures have been generated for each of the models and predefined textures are loaded for directories and a back button of each subdirectory. These icons are a folder and an arrow pointing to the left respectively.

By hovering over an item, text is displayed with the model's name; when the item is selected the model file is loaded and attached to the users wand so that it can be positioned in the environment. Any number of models and duplicates can be loaded into the environment.

\subsection{The Virtual Keyboard}

This application shows the visibility of the gMenu system by creating a full keyboard layout in front of the user, enabling text input while in a virtual environment Fig,2(a),

The user can type each letter by moving their hand to collide with the appropriate button. It would be possible to "two finger type" using this gMenu keyboard if two hand trackers were used within the environment. 


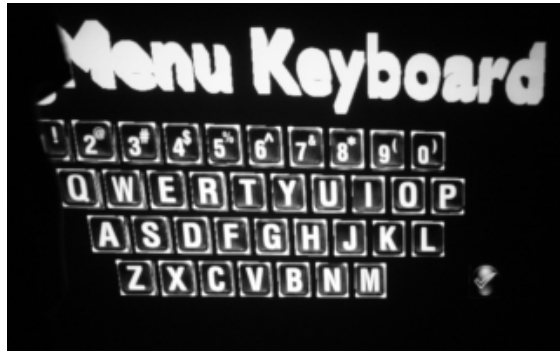

(a) gMenu Keyboard

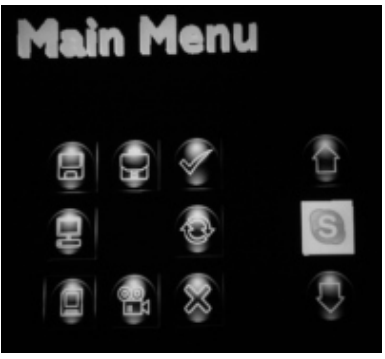

(b) Custom Item Layout

Fig. 2. Examples of the gMenu system in use

The keyboard can be very useful for naming files, text messaging other users within networked environments or debugging applications still under development while still within the environment. This could be achieved by generating a virtual terminal.

\subsection{Environment Controller}

This application combines a file browser with other control options to enable users within a virtual environment to load, edit, and save scenes without having to leave the virtual reality system. Scenes are loaded in the same way as the 3D model viewer, but the scene objects can be selected bring up a control menu to perform simple manipulations of the objects. The scene can then be saved and closed.

Scenes that incorporate audio streams allow extra options in the control menu to change the volume within the environment.

\section{Conclusion and Future Work}

The gMenu system is very easy to create as a programmer and customise as a user. Because of these abilities it is very easy to simulate many types of 3D menus that have already been developed and researched, and will also enable quick prototype designs of new new menu ideas to be produced and tested in the future.

There has been positive reactions towards usability from the initial tests and applications created using the gMenu system. It shows potential to be used in multiple application tasks with its ability to be highly customisable and styled. Future user testing has been planned for the gMenu system comparing its usefulness and usability with other menu systems.

With a fully customisable system like gMenu and the ability to control both a system and its environments brings us a step closer to some of the capabilities that are possible with desktop operating systems. 
There are plans to introduce a third dimension to the gMenu grid allowing developers more freedom with the menu shapes and positions, making the option to generate menus resembling the Command and Control Cube easier for example.

Customising and creating new menus live within the environment by adding and removing items from menu grid to grid, or even placing menu items into the environment on there own, are some ofthe features currently being developed.

There is also future plans to create more menu item types to be used in gMenu such as toggle buttons, passive indicators, analogue output selections, and dynamic items that can be created by the users.

\section{References}

1. Bowman, D.A., Kruijff, E., LaViola, J.J., Poupyrev, I.: 3D User Interfaces: Theory and Practice. Addison Wesley Longman Publishing Co., Inc., Redwood City (2004)

2. Haffegee, A.: VieGen: An Accessible Toolset for the Conguration and Control of Virtual Environments. Ph.D thesis, Centre for Advanced Computing and Emerging Technologies (ACET), School of Systems Engineering, University of Reading (2008)

3. Dachselt, R., Hbner, A.: Three-dimensional menus: A survey and taxonomy. Computers and Graphics 31(1), 53-65 (2007)

4. Bowman, D.A.: Interaction Techniques for Common Tasks in Immersive Virtual Environments. Ph.D thesis, Georgia Institute of Technology (1999)

5. Chan, C.N., Oe, S., Lin, C.S.: Active eye-tracking system by using quad ptz cameras. In: Industrial Electronics Society, 2007. IECON 2007. 33rd Annual Conference of the IEEE, pp. 2389-2394 (November 2007)

6. Jacoby, R.H., Ellis, S.R.: Using virtual menus in a virtual environment. In: Alexander, J.R. (ed.) Society of Photo-Optical Instrumentation Engineers (SPIE) Conference Series, vol. 1668, pp. 39-48 (June 1992)

7. Coninx, K., Van Reeth, F., Flerackers, E.: A hybrid 2d/3d user interface for immersive object modeling. In: Proceedings of Computer Graphics International, 1997, pp. 47-55 (June 1997)

8. Bowman, D.A., Wingrave, C.A.: Design and evaluation of menu systems for immersive virtual environments. In: VR 2001: Proceedings of the Virtual Reality 2001 Conference (VR 2001), Washington, DC, USA, p. 149. IEEE Computer Society, Los Alamitos (2001)

9. Wloka, M.M., Greenfield, E.: The virtual tricorder. Technical report, Department of Computer Science, Brown University (1995)

10. Gerber, D., Bechmann, D.: The spin menu: a menu system for virtual environments. In: Virtual Reality, 2005. Proceedings. VR 2005, pp. 271-272. IEEE, Los Alamitos (2005)

11. Grosjean, J., Burkhardt, J.M., Coquillart, S., Richard, P.: Evaluation of the command and control cube, p. 473. IEEE Computer Society, Los Alamitos (2002) 